\title{
RIGHT ALTERNATIVE RINGS OF CHARACTERISTIC TWO
}

\section{R. L. SAN SOUCIE}

1. Introduction. Right alternative rings have recently been investigated by Skornyakov, Kleinfeld, and the author. Skornyakov [3 $]^{1}$ showed that a right alternative division ring of characteristic not two is alternative. The author, in [2], extended this result by proving that a right alternative division ring of characteristic two is alternative if (and only if) it satisfies

$$
w(x y \cdot x)=(w x \cdot y) x
$$

for all $w, x, y$ and showed by example that (1.1) can fail to hold. Prior to this, Kleinfeld [1] generalized the Skornyakov theorem in another direction by assuming only the absence of one sort of nilpotent element. We now specify Kleinfeld's result in detail.

Let $F$ be the free nonassociative ring generated by $x_{1}$ and $x_{2}$ and suppose that $R$ is any right alternative ring. Kleinfeld calls $t, u, v$ in $R$ an alternative triple if (i) there exist elements $\alpha\left[x_{1}, x_{2}\right], \beta\left[x_{1}, x_{2}\right]$, $\gamma\left[x_{1}, x_{2}\right]$ in $F$ and elements $r_{1}, r_{2}$ in $R$ such that $t=\alpha\left[r_{1}, r_{2}\right], u=\beta\left[r_{1}, r_{2}\right]$, $v=\gamma\left[r_{1}, r_{2}\right]$ and (ii) if $s_{1}$ and $s_{2}$ are elements from an arbitrary alternative ring, and if $t^{\prime}=\alpha\left[s_{1}, s_{2}\right], u^{\prime}=\beta\left[s_{1}, s_{2}\right], v^{\prime}=\gamma\left[s_{1}, s_{2}\right]$, then $\left(t^{\prime}, u^{\prime}, v^{\prime}\right)$ $=0$. The ring $R$ is said to have property (P) if $t, u, v$ an alternative triple in $R$ and $(t, u, v)^{2}=0$ imply $(t, u, v)=0$. By the definition of an alternative triple, an alternative ring has property $(\mathrm{P})$. Kleinfeld's result is the converse, assuming characteristic not two; that is, a right alternative ring of characteristic not two is alternative if (and only if) it has property $(\mathrm{P})$.

We herein extend this line of investigation by proving that a right alternative ring of characteristic two, satisfying (1.1), is alternative if (and only if) it has property $(\mathrm{P})$. The methods are mainly those used in [2], coupled with two essential lemmas (numbered 4 and 5 in our paper) due to Kleinfeld. Following [2], we say that $R$ is strongly right alternative if $R$ is a right alternative ring satisfying (1.1). Throughout the paper, $R$ will always denote such a ring, with the additional hypothesis that $R$ have characteristic two.

2. Previous results. We begin with the following definition due to Skornyakov [3]. Let $a$ and $b$ be fixed elements of $R$. Then we shall

Presented to the Society, May 1, 1954; received by the editors October 12, 1954.

${ }^{1}$ Numbers in brackets refer to the bibliography at the end of the paper. 
denote by $u(a, b)$ the set of all elements $x$ in $R$ such that $x a \cdot b=x \cdot b a$. We observe that $u(a, b)$ is closed under addition.

Lемма 1. The following identities hold in $R$ :

$$
\begin{aligned}
(w, x, x y) & =(w, x, y) x \\
(w, x, y z)+(w, y, x z) & =(w, x, z) y+(w, y, z) x, \\
\left(w, x^{2}, y\right) & =(w, x,(x, y)), \\
((w, x, y), x, y) & =(w, x, y)(x, y), \\
(w x, y, z) & =w(x, y, z)+(w, y, z) x+(w, x,(y, z)) .
\end{aligned}
$$

LEMMA 2. $x$ is in $u(a, b)$ if and only if $(x, a, b)=x(a, b)$.

Lemma 3. If $x$ is in $u(a, b)$ and $x$ is in $u(a, b a)$, then $x(a, a, b)=0$.

Lemma $4 .(x, a, b)$ and $(x, a, b)$ are in $u(a, b)$.

Lemma 5. If both $y$ and $x y$ are in $u(a, b)$, then $(x, a, b) y=0$.

Proofs of Lemmas 1-3 may be found in [3], and proofs of Lemmas 4 and 5 in [1].

As in [2], we define the mapping $\pi$ (of $R$ into $R$ ) by $x \pi=(x, a, b)$, for fixed $a, b$ in $R$. Then (2.5) may be written

$$
(w x) \pi=w \cdot x \pi+w \pi \cdot x+(w, x,(a, b)) .
$$

3. The main theorem. We henceforth assume that $R$, a strongly right alternative ring of characteristic two, has property $(\mathrm{P})$.

Lemma 6. $(a, b)=0$ implies $(a, a, b)=0$.

Proof. Assuming $(a, b)=0$, we have that $(x a, a, b)=x(a, a, b)$ $+(x, a, b) a$, using (2.5). Lemma 4 can be invoked to show that $x(a, a, b)$ is in $u(a, b)$. But $(a, a, b)$ is also, so that, by Lemma 5 , $(x, a, b)(a, a, b)=0$. Setting $x=a$ and using property $(\mathrm{P})$ proves the lemma.

For convenience, we set $c=(a, a, b), d=(a, b)$, and $e=(d, a, d)$. This enables us to state

LEMma 7. (i) $d \pi=(d, a, b)=0$, $c d=d c$, (iv) $(c, c, d)=(d, d, c)=0$.

(ii) $(d, a, x)=((a, x), a, b)$, (iii)

Proof. (i), (ii), and (iii) are proved in [1, Lemmas 5 and 7] since Kleinfeld does not use the assumption on the characteristic until he reaches his equation (4). Then (iv) follows from Lemma 6.

LeMma 8. For arbitrary $u$ in $u(a, b),(d, c, u)=e u$. 
Proof. Observing that $u \pi=u d$, and $a \pi=c$, we compute $(d, a, u) \pi$ and obtain

$$
(d, a, u) \pi=(d, a, u) d+(d, c, u)+e u .
$$

However, Lemma 7 (ii) shows that $(d, a, u)$ is in $u(a, b)$ and thus the lemma follows from Lemma 2 and (3.1).

LemMa 9. $e=0$.

Proof. The first three sentences in the proof of Lemma 7 in [1] show that $e c=0$. Hence $(d e \cdot c) e=0$, using (1.1). We now apply Lemma 8 with $u=e$ and get $e^{2}=(d, e, c)$ so that $e^{2}=d e \cdot c$. Hence $e^{3}=0$, so $e^{4}=0$. But property (P) implies $e^{2}=0$, and, again, $e=0$.

Lemma 10. $(c, a, d)$ is in $u(a, b)$.

Proof. As in the proof of Lemma 8 , we compute $(x, a, d) \pi$ and obtain

$$
(x, a, d) \pi=(x, a, d) d+((x, a, b), a, d)+(x, c, d)+(x, a d, d) .
$$

Substituting $x=c$ in (3.2) gives $(c, a, d) \pi=(c, a, d) d+\theta$, where $\theta=(c d, a, d)+(c, d, a d)=c e+(c, a, d) d+(c, d,(a, d))+(c, d, a d)$. However, $(c, d, a d)=\left(c, a, d^{2}\right)+(c, a, d) d=(c, d,(a, d))+(c, a, d) d$, by (2.2) and (2.3). Hence $\theta=0$ and the proof is complete.

We can now prove our main result.

THEOREM. Let $R$ be a strongly right alternative ring of characteristic two. Then $R$ is alternative if and only if it has property $(\mathrm{P})$.

Proof. The necessity is obvious. For the sufficiency, we begin by showing that $c^{2}$ is in $u(a, b)$. Indeed, $(c a, a, b)=c^{2}+c d \cdot a+(c, a, d)$. However, Lemma 7 (ii) shows that $(d, a, c)$ is in $u(a, b)$. But $d \cdot c a$ is in so that $d c \cdot a=c d \cdot a$ is in, and $(c, a, d)$ is in by Lemma 10 . Hence $c^{2}$ is in $u(a, b)$. However $c$ is also in $u(a, b)$, and thus, using Lemma 5, $c d \cdot c=c^{2} d=0$. But $c d$ is in $u(a, b)$ and another application of Lemma 5 gives $(c d)^{2}=0$, from which $c d=0$.

$\operatorname{Now}^{3}((a, a, b), a, b)=c d=0$ and linearization yields $((a, a, x), a, b)$ $=(c, a, x)$. Put $x=a b$ and obtain that $(c a, a, b)=0$. This implies that $c^{2}=(c, a, b a)$ and therefore $c^{2}$ is in $u(a, b a)$. Lemma 3 yields $c^{3}=0$, from which $c^{4}=0$, and, using property $(\mathrm{P}), c^{2}=0, c=0$.

${ }^{2}$ It is an easy matter to verify that a strongly right alternative ring of arbitrary characteristic is power-associative. Therefore, powers of a single element are welldefined and we may write $e^{3}, e^{4}$, etc. without ambiguity.

${ }^{3}$ The last paragraph of our proof is the same as that in [1], but we repeat the few lines here for completeness. 


\section{BIBLIOGRAPHY}

1. Erwin Kleinfeld, Right alternative rings, Proc. Amer. Math. Soc. vol. 4 (1953) pp. 939-944.

2. R. L. San Soucie, Right alternative division rings of characteristic two, Proc. Amer. Math. Soc. vol. 6 (1955) pp. 291-296.

3. L. A. Skornyakov, Right alternative fields, Izvestia Akad. Nauk SSSR Ser. Mat. vol. 15 (1951) pp. 177-184.

The University of Oregon

\section{ON AN ITERATIVE PROCEDURE FOR OBTAINING THE PERRON ROOT OF A POSITIVE MATRIX}

\section{RICHARD BELLMAN}

1. Introduction. The purpose of this paper is to present a new iterative procedure for obtaining the characteristic root of largest absolute value of a positive matrix.

The origin of the method is as follows. There is a result of von Neumann [7], a generalization of his fundamental min-max theorem in the theory of games [8], to the effect that

$$
\operatorname{Min}_{y} \operatorname{Max}_{x} \frac{(x, A y)}{(x, B y)}=\underset{x}{\operatorname{Max}} \underset{y}{\operatorname{Min}} \frac{(x, A y)}{(x, B y)}
$$

where the variation is over the region defined by

$$
R \text { : }
$$

$$
\text { (a) } x_{i} \geqq 0, \quad \sum_{i=1}^{n} x_{i}=1,
$$

$$
\text { (b) } y_{i} \geqq 0, \quad \sum_{i=1}^{n} y_{i}=1 \text {, }
$$

and it is assumed that $B$ has the property that

$$
(x, B y) \geqq b>0
$$

for all $(x, y) \in R$.

It was observed by Shapley [6] that this result can be obtained as a by-product of the theory of "games of survival," cf. $[1 ; \mathbf{5} ; \mathbf{6}]$, which requires only the fundamental min-max theorem, by considering the equation for $\lambda$,

Received by the editors November 22, 1954. 